\title{
Contenir et prévenir la violence. La discipline scolaire et universitaire sous l'Ancien Régime (XVIIe-XVIIIe siècles)
}

Containing and preventing violence. School and university discipline under the French Ancien Régime (17th-18th centuries)

Gewalt zügeln und ihr vorbeugen. Disziplin in Schule und Universität im Ancien Régime (17.-18. Jahrhundert)

Contener y prevenir la violencia. La disciplina escolar y universitaria bajo el Antiguo Régimen (siglos XVII-XVIII)

\section{Laurence Brockliss}

Traducteur : Annie Bruter

\section{(2) OpenEdition}

Journals

Édition électronique

URL : https://journals.openedition.org/histoire-education/539

DOI : 10.4000/histoire-education.539

ISSN : 2102-5452

Éditeur

ENS Éditions

\section{Édition imprimée}

Date de publication : 1 avril 2008

Pagination : 51-66

ISBN : 978-2-7342-1117-4

ISSN : 0221-6280

Référence électronique

Laurence Brockliss, « Contenir et prévenir la violence. La discipline scolaire et universitaire sous l'Ancien Régime (XVIIe-XVIIIe siècles) », Histoire de l'éducation [En ligne], 118| 2008, mis en ligne le 01 janvier 2013, consulté le 20 mai 2021. URL : http://journals.openedition.org/histoire-education/539 ; DOI : https://doi.org/10.4000/histoire-education.539 


\title{
Contenir et prévenir la violence
}

\section{La discipline scolaire et universitaire sous l'Ancien Régime (XVII $^{e}$-XVIII ${ }^{e}$ siècles $)^{1}$}

\author{
Laurence W. B. BROCKLISS
}

À la fin du Moyen Âge, les élèves et les étudiants sont connus pour être indisciplinés et violents. Comme ils forment souvent de petites bandes autonomes vivant de rapines et migrant de ville en ville, leur présence a ses avantages, mais aussi ses inconvénients. L'existence d'une université dans une ville est favorable au commerce et la fait connaître à l'étranger, mais induit une rivalité entre town et gown ${ }^{2}$ qui dégénère parfois en combats de rue aux conséquences mortelles. Il semble cependant que la situation commence à s'améliorer à partir du début du $\mathrm{XVII}^{\mathrm{e}}$ siècle. La tolérance des autorités envers les associations estudiantines prend fin (de même qu'à l'égard des associations de jeunes en général), une discipline plus stricte et aux procédés plus imaginatifs est imposée dans les classes et on enseigne aux jeunes les vertus du self-control et de la politesse, tout cela dans le cadre plus vaste de la campagne d'acculturation que Norbert Elias a appelée le "processus de civilisation " ${ }^{3}$. Dans sa description classique de la socialisation des élèves sous l'Ancien Régime, Philippe Ariès affirmait, en 1960, qu'on peut

1 Traduit de l'anglais par Annie Bruter.

2 Town and gown : cette expression anglaise, dont le sens littéral est " la ville et la robe ", est passée en français pour désigner une ville et son université.

3 Norbert Elias, The Court Society, trad. anglaise Dublin, 2006 (trad. fr. La Société de cour, Paris, Flammarion, 1985). Sur la critique de la subculture de la jeunesse à la fin du Moyen Âge, voir Giovanni Levi and Jean-Claude Schmitt (eds.), A History of Young People in the West, trad. anglaise Cambridge, Mass., 1997, 2 vol. (trad. fr. Histoire des jeunes en Occident, Paris, Seuil, 1996, 2 vol.). 
percevoir un changement radical dans la conduite des adolescents en France à partir du milieu du XVII e siècle : avant cette date, les nouvelles normes prônées par les ouvrages pédagogiques des humanistes de la Renaissance et de leurs successeurs de la Contre-Réforme n'ont qu'un effet limité ; au XVIII ${ }^{e}$ siècle, en revanche, la classe est devenue un lieu beaucoup plus paisible et ordonné, où l'autorité du maître est largement reconnue et ses élèves raisonnablement studieux ${ }^{4}$. La description de P. Ariès ne reposait toutefois que sur une documentation limitée. Le présent article confronte cette vision à celle qui émane de la documentation plus riche qui était disponible à l'époque où j'ai fait paraître une étude de l'enseignement supérieur français, une trentaine d'années plus tard ${ }^{5}$. Il examine la conduite de deux groupes différents de jeunes : tout d'abord, celle des " artiens ", âgés de dix à dix-huit ans, qui fréquentent les 350 collèges enseignant les humanités latines et grecques et aussi, pour un petit nombre d'entre eux, la philosophie ; ensuite, celle de leurs aînés qui étudient la théologie, le droit et la médecine dans la vingtaine d'universités françaises. Sont toutefois exclus de l'étude les nombreux pensionnats et institutions privées qui sont apparus au cours du XVIII ${ }^{\mathrm{e}}$ siècle ${ }^{6}$.

\section{I - Discipline des élèves, indiscipline des étudiants}

Guel que soit le mode d'enseignement des arts ou des sciences aux XVII et XVIII ${ }^{e}$ siècles, la conduite attendue des élèves ou des étudiants en classe est toujours la même. Toutes les sources, très diverses, émanant de cette époque en donnent une image convergente, dont les traits essentiels sont les suivants. L'étudiant modèle est assidu, tout à la fois en classe et à la chapelle, arrive à l'heure aux cours et ne les quitte que quand la cloche retentit. De plus, il y vient habillé correctement et muni du matériel nécessaire : il porte la sobre robe universitaire de la couleur qui correspond à son statut, il ne porte jamais d'épée ni d'arme offensive

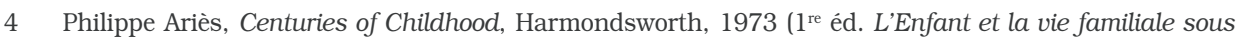
l'Ancien Régime, Paris, Plon, 1960), chap. 10 et 13.

5 Laurence W. B. Brockliss, French Higher Education in the Seventeenth and Eighteenth Centuries: A Cultural History, Oxford, Clarendon Press, 1987. La partie centrale de cet article est la traduction en français des p. 61-70.

6 La fin de l'enseignement des arts dans les universités et la naissance des collèges sont bien décrits dans Roger Chartier, Marie-Madeleine Compère, Dominique Julia, L'Éducation en France du XVI ${ }^{e}$ au XVIII siècle, Paris, SEDES, 1976, chap. 5. Pour les collèges pris individuellement, voir Marie-Madeleine Compère, Dominique Julia, Les Collèges français. 16e-18e siècles, Paris, INRP, 1984-2002, 3 vol. Sur les institutions nouvelles du XVIII ${ }^{e}$ siècle, voir Philippe Marchand, "Un modèle éducatif original à la veille de la Révolution ", Revue d'histoire moderne et contemporaine, 22, 1975, p. 549-567. 
et il n'oublie jamais son écritoire, ses plumes et son papier. En classe, il fait tout ce qui est exigé de lui : il garde le silence, sauf si on s'adresse personnellement à lui, et ne se dispute évidemment jamais avec les autres élèves ; lorsqu'il prend la parole, il s'adresse au professeur avec déférence et, s'il est interrogé, répond toujours en latin. Il est en permanence modeste et attentif. En outre, si c'est un artien, il vient en classe en ayant fait le devoir donné le jour précédent. En d'autres termes, l'élève modèle est un parangon de vertu ${ }^{7}$.

Dans quelle mesure cet idéal est-il conforme à la réalité ? Parmi les artiens, et les collégiens en général, le degré de corrélation entre les deux semble d'un niveau raisonnable, en particulier au fur et à mesure qu'on avance dans le temps. Des deux siècles dont il est question ici nous sont parvenus de nombreux témoignages d'actes individuels d'indiscipline, mais les sources suggérant que l'inconduite aurait été le fait de plus qu'une petite minorité chez les collégiens et les séminaristes sont peu nombreuses ${ }^{8}$. En outre, on ne peut guère accuser l'élève indiscipliné de brutalité. Les agressions contre les professeurs ne sont certes pas inconnues, mais on ne peut pas dire qu'il s'agisse de faits banals. Il en va de même des grèves du genre de celle qu'organise Marmontel à Mauriac, dans les années 1740, après avoir réussi à convaincre ses camarades rhétoriciens que son refus de recevoir le fouet est justifié ${ }^{9}$. Normalement, les élèves indisciplinés

7 Nombreuses sources, par exemple Ernest Gaullieur, Histoire du collège de Guyenne, Paris, Sandoz et Fischbacher, 1874, p. 106-107 : description de l'élève idéal selon Gouvéa (l'un des premiers principaux du collège de Guyenne) ; Ratio atque institutio studiorum Societatis Jesu, auctoritate septimae congregationis generalis aucta, Rome, 1616, p. 18-19 ; Réformation de l'Université de Paris, Paris, imp. de J. Mettayer et P. L'Huillier, 1601, p. 19-20 et 129-130 ; Jean Grangier, De l'état du collège de Dormans dit Beauvais, 1628, Bibliothèque de la Sorbonne, U 9, p. 40-44. Cette image ne diffère guère de celle qu'avaient présentée Érasme et Vivès ; elle avait été popularisée au XVI siècle par les dialogues de Mathurin Cordier, lui-même professeur à Paris. On la trouve encore à la fin de la période dans un texte édifiant : voir abbé L. B. Proyart, L'Écolier vertueux, ou vie édifiante d'un écolier de l'Université de Paris, mort le 23 décembre de l'année 1768, Paris, Berton, 1772, p. 38-44.

$8 \quad C f$. les rapports sur le collège jésuite de Caen à la fin du XVII ${ }^{e}$ siècle et sur le collège bénédictin de Sorèze au milieu du XVIII enalysés dans Jean de Viguerie, L'Institution des enfants : l'éducation en France, XVI ${ }^{e}$-XVIII ${ }^{e}$ siècles, Paris, Calmann-Lévy, 1978, p. 240. Au collège du Mont de Caen, $60 \%$ des élèves de rhétorique de 1677 avaient de bonnes mœurs (boni mores) : BNF, ms L. 10990, f ${ }^{\text {os }} 71-85$. Les séminaristes pouvaient être de véritables parangons de vertu. Selon Baston, les élèves de philosophie qu'il avait à Angers étaient si sages qu'il pouvait dicter son cours à travers les fenêtres de la classe tout en se promenant dans le jardin : Mémoires de l'abbé Baston, éd. Julien Loth et Ch. Verger, Paris, Alphonse Picard et fils, 1897, t. 1, p. 160

9 Jean de Viguerie, L'Institution..., op. cit., p. 246 ; Philippe Ariès, op. cit., p. 303 ; Jean-François Marmontel, Mémoires, éd. John Renwick, Clermont-Ferrand, G. de Bussac, 1972, t. 2, p. 12-17. Les élèves, cependant, ne manquaient pas totalement de réaction devant ce qu'ils percevaient comme une injustice ; il doit y avoir eu de nombreux incidents semblables à celui qui eut lieu au collège oratorien de Nantes en 1788 , où des élèves de troisième en colère lancèrent des pierres aux fenêtres du collège après qu'un de leurs condisciples eut été puni injustement : Archives municipales (désormais AM) de Nantes, GG 661, non paginé, rapport du 26 février. 
ne se rendent coupables que de délits mineurs. Le collégien d'Ancien Régime peut manquer la classe, il peut oublier continuellement son écritoire, faire le devoir d'un autre à sa place ou lire un roman caché par ses livres de classe, mais ce n'est pas un voyou. Au pire, il peut ameuter la cour et essayer d'entrer en classe de force avant que la cloche ait sonné, comme le font à deux reprises des élèves d'Amiens, en 1769 et en $1784^{10}$.

$\mathrm{Si}$, au cours de la période, il y a eu un moment et un lieu où les collégiens se sont livrés à des débordements, c'est dans l'Université de Paris au début du XVII ${ }^{\mathrm{e}}$ siècle. À cette époque, il semble que le collégien ordinaire, à Paris, ait fait peu de cas des règlements de l'Université et des collèges. Selon un écrit du principal du collège de Beauvais, Jean Grangier, daté de 1628, il régnait partout un tel chaos que ses efforts pour améliorer la situation dans l'établissement après sa nomination, en 1615, n'avaient que partiellement réussi. Si un élève était trop sévèrement puni, il s'en allait tout simplement ailleurs. Du coup, les collégiens de Beauvais restaient bruyants et impolis. Mais le plus grand problème de Grangier était l'assiduité. Ce n'est pas seulement que les élèves manquaient des cours. Comme ils ne faisaient pas leurs devoirs, ils n'arrivaient en classe qu'une fois passé le moment de la correction. Et comme ils étaient rares à vouloir étudier le grec, ils quittaient la classe chaque fois qu'il y était question de cette langue ${ }^{11}$.

La situation dans les collèges parisiens doit toutefois s'être améliorée au cours des années 1630 car une enquête universitaire sur la discipline menée en 1642-43 trouve peu à redire, et les critiques d'ensemble disparaissent après cette date $^{12}$. En conséquence, si l'on rencontre des problèmes de discipline dans la seconde moitié du XVII ${ }^{e}$ siècle et au XVIII ${ }^{e}$ siècle, ils sont le fait des étudiants

10 Charles Bailey, "French Secondary Education 1763-1790: The Secularization of Ex-Jesuit Colleges", Transactions of the American Philosophical Society, 68, 6 (1978), p. 94. Dans la liste des délits mineurs, on trouve les péchés véniels fréquemment condamnés par les règlements de collège. L'oubli de l'écritoire semble avoir été fréquent : à Montpellier, à partir de 1626, les élèves qui oubliaient leur matériel devaient être battus, $c f$. Alexandre-Charles Germain, La Faculté des arts et l'ancien collège de Montpellier 1242-1789, Montpellier, impr. de Boehm et fils, 1882, p. 40-41. Le problème de l'absentéisme ne doit pas être exagéré. Si le frère de Pierre Bayle, Joseph, se plaignait que les trois quarts de sa classe de philosophie étaient souvent absents à l'académie protestante de Puylaurens (auparavant sise à Montauban) au cours des années 1670, un absentéisme aussi marqué n'était pas habituel. À la même date, dans la classe de quatrième du collège jésuite de Caen, seuls 28 élèves sur 134 étaient notés comme fréquemment absents, et pas plus de 8 comme l'étant presque en permanence : voir Élisabeth Labrousse, Pierre Bayle, La Haye, M. Nijhoff, 1963, t. 1, p. 60-61 ; BNF, ms L. 10990, fos 22-36.

11 Jean Grangier, op. cit., p. 40-50 et 79-80. Sur le " hooliganisme " des étudiants de Paris au début du XVII ${ }^{\mathrm{e}}$ siècle, voir aussi Réponse d'un étudiant en l'Université de Paris à un sien ami (1616), brochure imprimée anonyme dans BNF, ms fr. 15783, p. 64.

12 Archives de l'Université de Paris, registre 26, fos 313-318, inspection du 21 octobre 1642 au 5 févier 1643. 
des facultés supérieures, non celui des collégiens. Il est vrai que les données touchant la conduite des étudiants sont moins faciles à trouver, mais celles qui sont disponibles vont à l'appui de l'idée qu'un grand nombre de ceux-ci se sont montrés turbulents et irrévérencieux pendant toute la période, y compris parmi les étudiants en théologie. Sans doute ces derniers ne lancent-ils pas de boules de neige à leurs professeurs ou ne viennent-ils pas en cours avec leur épée, comme les étudiants en droit ont la réputation de le faire, mais ce sont des bavards invétérés. Selon Baston, ils ne sont attentifs que lorsque le professeur dicte le cours ; une fois le cours dicté et l'explication commencée, toute discipline s'évanouit. Seuls quelques étudiants vertueux, comme Baston et ses camarades de Saint-Sulpice, prennent la peine d'écouter. Les autres se promènent à l'extérieur s'il fait beau, ou se regroupent dans les coins et parlent si fort que le professeur ne s'entend pas lui-même. Ce dernier n'a aucun moyen de faire cesser le bavardage :

Il avait beau frapper de la main sur son carton, ou de son carton sur les bords de la chaire ; beau se plaindre, prendre de l'humeur, menacer ; on s'arrêtait, mais pour rire aux éclats, si la semonce paraissait plaisante, ou l'était effectivement, et aussitôt le bourdonnement reprenait et continuait de plus belle. J'ai vu des professeurs prier ces babillards de se retirer, le leur demander en grâce, leur donner congé et ne rien obtenir ; cette jeunesse était scrupuleuse et voulait faire sa Sorbonne en conscience $^{13}$.

Quels que soient les défauts des étudiants en médecine et en théologie, du moins assistent-ils aux cours. S'ils manquent quelque chose, ce sont les disputes orales que doivent mener les candidats à un diplôme et auxquelles les étudiants plus jeunes sont censés assister ${ }^{14}$. Les étudiants en droit, en revanche, ne sont pas seulement turbulents en cours ; surtout, ils mettent de la mauvaise

13 Baston, op. cit., t. 1, p. 115-116 ; pour une description analogue, voir Thomas-Jean Pichon, Des études théologiques, ou Recherches sur les abus qui s'opposent au progrés de la théologie dans les écoles publiques, \& sur les moyens possibles de les réformer en France, Avignon, Pierre Vente, 1767, p. 19-21. À Bourges, en revanche, il semble que les théologiens disparaissent purement et simplement quand l'explication commence : $c f$. Bibliothèque municipale (dorénavant BM) de Bourges, BY 20391 (7), "Compte rendu aux chambres assemblées " (1764), p. 205-206. Les plaintes au sujet de la conduite des étudiants en droit sont légion : bombarder les professeurs de boules de neige ou de pois ou de cailloux est une spécialité parisienne de la fin du XVII ${ }^{e}$ siècle : voir BNF, ms fr. 21735, fos 100-103. On ne sait malheureusement rien de la façon dont se conduisent les étudiants en médecine.

14 Selon Baston, op. cit., t. 1, p. 87, les étudiants en théologie riches préfèrent payer une amende plutôt que de venir aux disputes. En 1753, les étudiants de Montpellier sont accusés de ne pas aller aux " exercices ordinaires "; la même année, ils organisent également un boycott des cours, mais cela semble avoir été une chose exceptionnelle, due au renvoi des représentants étudiants du conseil exécutif de la faculté : Alexandre-Charles Germain, L'École de médecine de Montpellier, ses origines, sa constitution, son enseignement, étude historique d'après les documents originaux, Montpellier, J. Martel aîné, 1880, p. 105-109. 
volonté à y assister. En fait, jusqu'en 1679, on se plaint continuellement que beaucoup de diplômés en droit n'ont jamais mis les pieds à la faculté avant de venir y chercher leur diplôme ; ils préfèrent rester chez eux et étudier par eux-mêmes ${ }^{15}$. Les témoignages à l'appui de ces accusations ne manquent pas. L'écrivain Charles Perrault n'est que l'un des nombreux Parisiens qui, au milieu du XVII ${ }^{e}$ siècle, vont chercher leur diplôme de droit à Orléans sans avoir jamais assisté aux cours ${ }^{16}$. Après 1679, toutefois, un absentéisme aussi ouvert devient virtuellement impossible puisque, lors de la réforme générale des études de droit qui prend place cette année-là, Louis XIV insiste sur le fait que les étudiants ne doivent pas être autorisés à obtenir leur diplôme s'ils n'ont pas signé le registre d'inscription au moins quatre fois dans l'année. Pour renforcer cette obligation, il est précisé qu'aucun diplômé ne sera reçu avocat si on ne trouve pas trace de cette inscription dans la copie du registre qui doit être conservée au présidial local. Après cela, les étudiants en droit ont sans aucun doute dû faire au moins des apparitions dans la faculté avant d'obtenir leur diplôme.

Mais ce que la réforme louisquatorzienne ne pouvait pas les obliger à faire, c'est à suivre les cours. On se plaint fréquemment, au XVIII siècle, des étudiants qui se contentent de s'inscrire chaque trimestre et payent un copiste pour suivre les cours à leur place. Selon Besnard, les étudiants en droit d'Angers sont si paresseux qu'ils ne viennent pas à l'appel quotidien, même quand on y distribue des places de théâtre gratuites. S'il faut en croire une ordonnance du bailliage de Caen, ils se laissent au contraire entraîner à mener une vie de plaisirs :

Un grand nombre de jeunes gens passent dans les billards la majeure partie d'un temps destiné aux classes [...] ils forment dans ces maisons des liaisons dangereuses,

15 La plainte la plus ancienne à laquelle on puisse assigner une date précise se trouve dans un mémorandum de 1615 attribué au professeur de droit Maran, de Toulouse : voir " Remontrance de la nécessité de rétablir les universités pour le rétablissement de l'État et des moyens de ce faire ", BNF, ms. L. 9948, fo 201 (imprimé). Autres exemples typiques : " Mémoire pour les anciens docteurs non régents de la faculté de décret [de Paris] ", BNF, ms fr. 18828, fos 3-9 (milieu du XVII ${ }^{\mathrm{e}}$ siècle , imprimé) ; Archives nationales (désormais AN), MM 153, fos 441-444, lettre au frère de Louis XIV sur la situation à Orléans, c. 1650 ; Fr. de Roye (professeur à Angers), Apologeticus pro omnibus Galliarum antecessoribus... (1665), p. 86-88, in BNF, ms fr. 18828, f 56 et seq. (imprimé).

16 Charles Perrault, Mémoires de ma vie..., éd. Paul Bonnefon, Paris, 1909, p. 29-30. Voir aussi Jean Rou, Mémoires inédits et opuscules, éd. Francis Waddington, Paris, Société pour l'histoire du protestantisme français, 1857, p. 30-32 ; N.-J. Foucault, Mémoires, éd. F. Baudry, Paris, Imprimerie impériale, 1862, p. 5. Les études de droit de Rou s'étaient limitées à une année d'assistance à des répétitions privées tenues chez le parlementaire parisien Le Coq. 
y prennent le goût de la dissipation et du libertinage, et trompent absolument l'espoir de leurs familles ${ }^{17}$.

De telles jérémiades contiennent un grain de vérité, comme l'attestent les mémoires de l'académicien Charles Pinot Duclos, qui reconnaît avoir été d'une incorrigible oisiveté pendant ses études : s'étant inscrit en droit à Toulouse pour la première fois en 1725, il s'absente aussitôt pour prendre des leçons auprès d'un maître d'armes. Même de futurs éminents juristes semblent prendre leurs études assez peu au sérieux. Le parlementaire Hénault ne fait aucune mention de ses études à la faculté de Paris dans ses Mémoires : il est significatif que ce soit seulement quand il devient président de la première chambre des enquêtes, en 1710, qu'il se mette sérieusement à l'étude du droit ${ }^{18}$.

\section{II - Des collèges mieux armés que les facultés}

Il n'est pas surprenant que la discipline soit bien meilleure parmi les collégiens que parmi les étudiants, car les autorités du collège disposent d'une vaste panoplie de sanctions et d'armes psychologiques pour contrôler et éradiquer la mauvaise conduite, en particulier chez les élèves d'humanités, qui fait défaut aux conseils des facultés. Comme la majeure partie de la pédagogie des collèges aux XVII et XVIII ${ }^{\mathrm{e}}$ siècles, ces armes ne sont pas neuves mais ont été forgées par les Frères de la vie commune, puis par leurs successeurs humanistes.

Tout d'abord, on décourage les atteintes à la discipline dans les classes en faisant en sorte que les délinquants soient repérés et les problèmes anticipés dans la mesure du possible. On y parvient grâce à un système élaboré de surveillance. La première ligne de défense est assurée par le portier, dont le rôle est de surveiller l'entrée pendant les heures de classe, de façon à délester les jeunes godelureaux de leur épée, à refuser l'entrée aux fauteurs de trouble potentiels

17 François-Yves Besnard, Souvenirs d'un nonagénaire, éd. Célestin Port, Paris, Champion, 1880 , p. 194 ; Alfred de Curzon, L'Enseignement du droit français dans les universités de France aux XVII et XVIII ${ }^{e}$ siècles, Paris, L. Tenin, 1920, p. 89. Pour des exemples variés de plaintes au sujet d'une assiduité insuffisante ou par procuration, voir ibid., p. 95-98; Marie-Antoinette Lemasne-Desjobert, La Faculté de droit de Paris aux XVII et XVIII ${ }^{e}$ siècle s, Paris, Cujas, 1966, p. 139-154 ; J. Barbot, Les Chroniques de la faculté de médecine de Toulouse du XIII e au XIX ${ }^{e}$ siècle, Toulouse, 1905, t. 1, p. 288-289.

18 Patrick Ferté, "L'Université de Toulouse aux XVII ${ }^{\mathrm{e}}$ et XVIII ${ }^{\mathrm{e}}$ siècle s : étude quantitative de la population étudiante de ses trois facultés supérieures de 1679 à la Révolution ", thèse de $3^{e}$ cycle, Université de Toulouse-Le Mirail, 1978, t. 1, p. 181 ; Charles-Jean-François Hénault, Mémoires, éd. François Rousseau, Paris, Hachette, 1911, p. 33-34. Pour une autre déclaration selon laquelle les études de droit ne commençaient qu'avec l'obtention du diplôme, voir Louis-Marie de La Revellière-Lépeaux, Mémoires, Paris, J. Hetzel, 1873, t. 1, p. 20-27 (il fut étudiant en droit à Angers). 
et à noter le nom des retardataires ${ }^{19}$. À l'intérieur de l'établissement, l'action du portier reçoit souvent le renfort de celle d'un membre de l'administration - appelé préfet des études chez les Jésuites - qui patrouille dans le bâtiment pour empêcher les élèves de quitter leur salle de classe ${ }^{20}$. Mais une grande partie du travail de surveillance est effectuée à l'intérieur de la classe par les élèves eux-mêmes. Dans chaque classe est choisi parmi eux un normateur dont le travail est de tenir le registre quotidien des présences. D'autres élèves (un par décurie, là où existe ce système) jouent le rôle de gardiens de la morale. Ils sont habituellement appelés censores (censeurs) et leur tâche consiste à rapporter au professeur toute manifestation d'indiscipline. Dans les collèges jésuites, les parents eux-mêmes sont encouragés à participer à cette œuvre de bien. La Ratio jésuite stipule que les élèves qui manquent la classe doivent revenir avec un mot de leurs parents donnant le motif de leur absence ${ }^{21}$.

En second lieu, l'indiscipline chez les artiens est contenue par l'effort pour rendre les études intéressantes. Telle est manifestement, en partie, la raison d'être du programme, beaucoup plus orienté vers la pratique que celui des facultés. Se fondant sur l'idée que les jeunes enfants ont bien plus de mal à soutenir leur attention que les adultes, on fait de la variété un ingrédient essentiel du maintien de la paix en classe. Mais on cherche à accentuer encore l'intérêt pour les études en encourageant l'esprit de compétition. Dès leur entrée dans la première classe de grammaire, les artiens apprennent que l'attention et le travail peuvent rapporter récompenses et honneurs. Chaque semaine, les élèves les plus sages et les plus zélés peuvent être récompensés par la permission de ne pas faire l'un

19 Le portier apparaît pour la première fois dans les collèges français au milieu du XVI siècle, par exemple au collège de Guyenne en 1547, au collège de l'Esquille (à Toulouse) en 1560, au collège de Beauvais (à Paris) en 1587 : cf. Ernest Gaullieur, op. cit., p. 220 ; R. Corrazé, "L'Esquille, collège des capitouls, 1550-1654 ", Mémoires de l'Académie des Sciences, Inscriptions et Belles-Lettres de Toulouse, $12^{e}$ série, $\mathrm{n}^{\circ} 15,1937$, et $\mathrm{n}^{\circ} 16,1938$, p. 179-180; AN, $\mathrm{H}^{3} 4785$ (27), rapports des collèges pour frais extraordinaires, 1587. Néanmoins, le potentiel offert par sa présence n’a pas été pleinement exploité tout de suite dans tous les cas. Au collège de Beauvais, le portier ne doit rester à son poste en permanence qu'à partir de 1626 ; avant cette date, il n'a qu'à ouvrir les portes le matin et les fermer le soir et est autorisé à mener ses propres études entre temps : $c f$. Marie-Dominique Chapotin (le P.), Une page de l'histoire du vieux Paris. Le collège de Dormans-Beauvais..., Paris, Durand et Pedone Lauriel, 1870, p. 235. Parmi les collèges de plein exercice inspectés par la commission de l'Université en 1642-1643, seul le collège du Plessis n'avait pas de portier et le principal déclara pour sa défense qu'il venait d'en embaucher un huit jours auparavant.

20 Ratio studiorum..., op. cit., p. 23-30. À Paris, cet officier était appelé le sous-maitre : cf. le règlement du collège de Beauvais de 1646, BNF, ms fr. 18828, fos 762-768. Au collège de Guyenne, en 1762, cette tâche était assurée par le principal : $c f$. Ernest Gaullieur, op. cit., p. 504.

21 Ratio studiorum, op. cit., p. 8-9. Un célèbre censeur chez les Jésuites est Marmontel : Mémoires, op. cit., t. 1, p. 14-15. À Toulouse, en 1564, le censeur était appelé " promoteur " ; à Paris, en 1601, " explorateur " : R. Corrazé, art. cit., p. 191 ; Réformation..., op. cit., p. 20. 
des devoirs du soir. Dans la plupart des collèges, il y a aussi des compositions mensuelles pour désigner le premier de la classe et le vainqueur reçoit un petit cadeau, tel qu'une croix en argent ${ }^{22}$. Surtout, presque partout, sont donnés des prix annuels récompensant le meilleur texte en prose ou la meilleure traduction de la composition de fin d'année. À Lille, on songe même, à la fin du XVIII ${ }^{\mathrm{e}}$ siècle, à instaurer un contrôle continu, tandis qu'à Paris, à partir de 1747, les meilleurs élèves de chaque classe ont en outre l'occasion de prendre part à un concours donnant droit à un prix de l'Université, le concours général ${ }^{23}$. Dans les collèges tenus par les congrégations enseignantes, en particulier par les Jésuites, l'esprit de compétition est poussé à l'extrême. Le système des décuries ne sert pas seulement à permettre au maître de se concentrer sur ses tâches les plus importantes, mais aussi à maintenir les élèves en alerte. Chaque membre de la décurie, du chef jusqu'au dernier de la troupe, est noté en fonction de son travail et apparié avec son homologue des décuries rivales. Les équipes concurrentes combattent pour la meilleure place presque quotidiennement, chaque membre de la décurie défendant l'honneur du groupe contre ses rivaux. Motif supplémentaire d'émulation, la composition mensuelle est l'occasion de revoir la hiérarchie interne de la décurie. Ces compositions deviennent de véritables champs de bataille où ceux qui se trouvent en tête peuvent tomber en disgrâce et ceux qui font partie du peloton parvenir au commandement. L'objectif ultime de l'élève est de gagner la

22 Il semble que ce soient les Jésuites qui permettent aux bons élèves de se dispenser de devoirs : $c f$. les commentaires du cahier d'Avignon de 1732, BM Avignon, ms 565, f ${ }^{\circ} 123$. Le régent parisien Rollin suggère d'user de récompenses particulières, telles qu'une visite à la manufacture de tapisserie des Gobelins, De la manière d'enseigner et d'étudier les belles-lettres, Paris, J. Estienne, 1726-1728, t. 4, p. 490. Pour un témoignage personnel sur la composition hebdomadaire ou mensuelle, voir François-Yves Besnard, op. cit., p. 55 ; Marmontel, op. cit., t. 1, p. 12.

23 Philippe Marchand, "L'émulation au collège de Lille 1765-1791 ", in Histoire de l'enseignement de 1610 à nos jours. Actes du 95e Congrès national des sociétés savantes, Reims, 1970, Paris, Bibliothèque nationale, 1974, p. 157-158 ; Charles Jourdain, Histoire de l'Université de Paris au XVII e et au XVIII ${ }^{e}$ siècle , Paris, Hachette, 1862, p. 381-385. Il y a des prix dans les collèges français tenus par les Jésuites à partir de 1558, mais aucun à Avignon et Paris avant 1571 et 1579 : voir François de Dainville, Les Jésuites et l'éducation de la société française. La naissance de l'humanisme moderne, Paris, Beauchesne et ses fils, 1940, p. 144-153 ; Marcel Chossat, Les Jésuites et leurs œuvres à Avignon, 1553-1768, Avignon, F. Seguin, 1896, p. 148 ; cette pratique est par la suite réglementée dans la Ratio studiorum, op. cit., p. 20. Ailleurs, la distribution des prix se met en place progressivement au cours de la période, par exemple à Montpellier à partir du début du XVII ${ }^{\mathrm{e}}$ siècle ; à Paris, au collège d'Harcourt, en 1643 ; au collège Mazarin dès son ouverture en 1690 ; au collège oratorien d'Angers en 1753 ; à partir de 1765 il y a des prix dans tous les collèges dépendant de l'Université de Paris : voir Alexandre-Charles Germain, La Faculté des arts..., op. cit., p. 24 ; BNF, ms fr. 4873, fos 4-9 ; AN, H3 2825, non fol., rapports sur les collèges ; Jacques Maillard, L'Oratoire à Angers aux XVII et XVIII ${ }^{e}$ siècle s, Paris, C. Klincksieck, 1975, p. 165 ; Charles Bailey, art. cit., p. 34. Si on en croit La Revellière-Lépeaux, op. cit., t. 1, p. 15, à Angers les prix n'étaient pas toujours donnés aux meilleurs élèves, mais plutôt à ceux de rang social élevé. 
compétition mensuelle pour prendre le titre d'Empereur, il capo dei capi ${ }^{24}$. Seuls les pédagogues de Port-Royal sont opposés à l'idée de compétition. Pour Pascal, celle-ci encourage la nonchalance, trait de caractère que l'éducation humaniste est censée combattre. Mais les maîtres de Port-Royal ont la chance d'avoir des classes réduites à quatre ou cinq élèves ${ }^{25}$.

Enfin, si la peur d'être pris et l'incitation à l'étude ne suffisent pas à empêcher la délinquance chez les élèves, les collèges peuvent toujours recourir à la sanction définitive qu'est l'exclusion. Dans les classes d'humanités, toutefois, c'est vraiment le dernier recours et on tente d'abord de remettre les élèves récalcitrants dans le droit chemin par une punition donnée au moment opportun. La littérature pédagogique de l'époque souligne sans relâche que les punitions ne doivent pas être arbitraires, mais fondées sur deux principes de base : tout d'abord, les professeurs doivent s'efforcer de blesser les élèves moralement plus que physiquement (Rollin pense que priver les élèves de leurs chaussures en classe constitue une punition adéquate pour les coupables de famille noble) ; ensuite, la sanction doit être proportionnelle au délit. Pris dans son sens littéral, ce principe fait qu'un élève en retard aura à décliner le mot pigritia (paresse) en latin et en grec. Mais surtout, il signifie que le fouet ou la canne doivent être employés avec modération, à titre d'ultime moyen de dissuasion. Rollin, par exemple, est convaincu que les châtiments corporels sont contre-productifs :

D'ailleurs ces châtiments donnent une aversion incurable pour des choses qu'on doit tâcher de lui faire aimer. Ils ne changent point l'humeur, et ne réforment point le naturel, mais le répriment seulement pour un temps, et ne servent qu'à éclater les passions avec plus de violence quand elles sont en liberté. Ils abrutissent souvent l'esprit, et l'endurcissent dans le mal ; car un enfant qui a assez peu d'honneur

24 François de Dainville, Les Jésuites et l'éducation..., op. cit., p. 144-153 ; Marchand, "L'émulation... ", art. cit., p. 153. Les compositions mensuelles déterminant la place et le rôle dans la classe sont déjà habituelles dans les collèges jésuites à la fin du XVI e siècle : voir les remarques dans L. de Richeome (alias Fr. de Montaigues), La Vérité défendue pour la religion catholique en la cause des Jésuites contre le plaidoyé d'Antoine Arnaud, Toulouse, veuve de J. Colomiez, 1595, p. 175 ; Étienne Tabourot, Les Bigarrures du seigneur des Accords, $4^{\mathrm{e}}$ livre, Paris, J. Richer, 1585, fos 20-21. Le président Hénault a souvent été Empereur à Louis-le-Grand : Mémoires, op. cit., p. 14.

25 Georges Snyders, La Pédagogie en France aux XVII et XVIII siècle s, Paris, Presses universitaires de France, 1965, p. 53-54. Cependant, la compétition n'est pas totalement rejetée. Coustel et Arnaud sont favorables à certains types de compétitions du moment que les moins bons n'en conçoivent pas de jalousie. Du Fossé, qui a été élève de Port-Royal, rapporte dans ses mémoires que les élèves rivalisaient en privé : voir H. C. Barnard, The Port-Royalists on education, Cambridge, 1918, p. 118-121. Un règlement pour le collège parisien de Montaigu en 1683 semble indiquer que d'autres pédagogues se montrent réservés à l'égard de certains aspects du système d'émulation, ici les prix, bien qu'on reconnaisse qu'ils incitent au travail : Michel Félibien, Histoire de la ville de Paris, Paris, G. Desprez, 1725, t. 5, p. 843. 
pour n'être pas sensible à la réprimande s'accoutume aux coups comme un esclave et se raidit contre la punition ${ }^{26}$.

Il est difficile de dire dans quelle mesure la pratique coïncide avec ce principe. Assurément, il existe des témoignages d'un usage excessif du fouet. Un certain nombre de sadiques ont laissé leur trace dans les archives, parmi lesquels un professeur de sixième au collège d'Harcourt à la fin de l'Ancien Régime, un certain M. L'Hermite, qui fouetta ses élèves parce qu'ils n'avaient pu pas rester une demi-heure dans la cour pleine de neige. La seule manière d'attendrir ce " cour de roche " était de le soudoyer " avec quelques livres de bougie, de chocolat, de sucre et de café offertes aux étrennes ". Mais de tels personnages sont probablement exceptionnels. La raillerie de Piron, selon laquelle les Jésuites ont été expulsés de France par des conseillers au Parlement à qui le fouet avait laissé des souvenirs cuisants, doit être prise pour un bon mot, et non pas pour parole d'Évangile. Les Jésuites s'efforcent de décourager le sadisme en interdisant au professeur d'administrer lui-même des corrections aux élèves et en chargeant de cette tâche un " fouetteur " officiel. Les Oratoriens sont également connus pour leur façon humaine de traiter les délinquants. De fait, au collège oratorien d'Angers, les châtiments corporels sont totalement bannis dans les années 1760, à la grande surprise de l'abbé Besnard, qui était habitué à voir le principal du collège séculier de Doué faire un fréquent usage du fouet, même à l'égard des élèves les plus âgés ${ }^{27}$.

Dans les facultés, en revanche, les autorités ne disposent d'aucune de ces armes. Si les professeurs ne peuvent pas capter l'attention par leur grande érudition ou leur charisme personnel, ils sont perdus ${ }^{28}$. Ils ne disposent pas du système de surveillance des collèges ; leur mode d'enseignement exclut le recours à

26 Rollin, op. cit., t. 4, p. 464. Pour les théories générales sur la punition, voir Ratio studiorum, op. cit., p. 109-110 ; Edmond Richer, Obstetrix animorum, Paris, A. Drovart, 1600, p. 49 ; Réformation, op. cit., p. 17 ; R. Corrazé, art. cit., p. 214-215 ; Rollin, op. cit., t. 4, p. 462-479 ; BM Avignon, ms 565, fos 122-124. Selon le registre contenant les rapports sur le collège jésuite du Mont de 1692, la Société n'excluait pas plus d'un ou deux élèves par classe chaque année : par exemple Archangelus Hamard, rhétoricien de mauvaise réputation, exclu "quod debitas poenas subire noluerit " : BNF, ms L. 10991, fo 22.

27 Camille de Rochemonteix, Un collège de Jésuites aux XVII e et XVIII siècle s, le collège Henri IV de La Flèche, Le Mans, Leguicheux, 1889, t. 2, p. 88 ; François-Yves Besnard, op. cit., p. 25 et p. 203. Dès 1725, les Oratoriens d'Angers reçoivent l'instruction de punir avec amour : Jacques Maillard, op. cit., p. 170-171. Parmi les sadiques, on peut compter un Jésuite que Baston a connu à Rouen et un curé qui a enseigné les rudiments du latin à La Revellière-Lépeaux ; il semble que les élèves du premier sont vengés quand le malheureux Jésuite est rossé dans la rue par une bande de jeunes conseillers au Parlement : Baston, op. cit., t. 1, p. 23-24 ; La Revellière-Lépeaux, op. cit., t. 1, p. 9.

28 Selon Baston, op. cit., t. 1, p. 116, un bon professeur peut se créer un public assidu assez nombreux pour réduire les fauteurs de trouble au silence. Quand un Chevreuil fait cours à la Sorbonne, il semble que les chahuteurs sortent ou limitent leurs propos à des chuchotements face à la centaine d'étudiants qui écoutent avec attention. 
la compétition ; et ils ne peuvent recourir aux mêmes punitions. De plus, la seule sanction qu'ils puissent infliger est d'efficacité limitée. Le candidat au premier diplôme que délivrent les facultés supérieures, c'est-à-dire le baccalauréat, doit obtenir de ses professeurs l'attestation qu'il a suivi leurs cours avec assiduité. Théoriquement, donc, les étudiants indisciplinés pourraient se voir refuser cette attestation. Mais un certain nombre de facteurs interviennent pour limiter cette possibilité. Tout d'abord, les étudiants ne cherchent pas tous à avoir un diplôme (en particulier parmi les étudiants en théologie) ; et, dans les grandes facultés, il est sans doute impossible aux professeurs d'identifier les étudiants absentéistes ou chahuteurs. De plus, la coutume est de délivrer les attestations sur la simple présentation du cahier sur lequel le cours a été transcrit, ce qui ne prouve nullement, comme on l'a vu, que le possesseur du cahier a suivi le cours en personne. Ainsi, l'unique moyen de pression dont disposent les professeurs est virtuellement inutile, ce qui explique sans nul doute pourquoi on tente, au XVIII ${ }^{e}$ siècle, de traiter le problème de l'absentéisme en recourant à des appels, quotidiens ou réguliers, dans un certain nombre de facultés, telle la faculté de théologie de Paris en $1734^{29}$. Non que ce procédé soit efficace dans des facultés plus grandes : les amis peuvent tout simplement répondre à la place des absents.

Finalement, en fait, la plupart des facultés laissent l'application des statuts à la charge de la juridiction civile. Cette dernière, toutefois, bien que de bonne volonté, n'est pas mieux placée pour traiter le problème. Elle peut menacer les délinquants de terribles amendes, mais on comprend qu'elle soit réticente à sévir quand ses fils ou ses connaissances font partie des fauteurs de trouble. Chaque année, la faculté de droit d'Angers rappelle aux étudiants que le présidial leur interdit de porter leur épée en cours. Mais ce règlement n'est jamais appliqué, et Besnard, qui est dans une autre faculté, se fiant à ce qu'il voit, passe le temps de ses études universitaires dans la conviction infondée que le port de l'épée est licite ${ }^{30}$.

29 Pierre Féret, La Faculté de théologie de Paris et ses docteurs les plus célèbres. Époque moderne, Paris, A. Picard et fils, 1900-1909, t. 6, p. 366-368 : la faculté ne dissimule pas les raisons pour lesquelles cette pratique a été introduite. Il y a aussi des appels dans les universités de Toulouse, Caen et Angers au XVIII ${ }^{\mathrm{e}}$ siècle. Au moins un registre d'appel tenu par le professeur nous est parvenu : AD Calvados, I D 556 (registre tenu par le professeur de théologie de Caen, Le Clerc de Beauberon, 1745-1780 : les absents sont notés).

30 François-Yves Besnard, op. cit., p. 123. Les arrêtés locaux contre le port de l'épée en cours sont légion : par exemple à Bourges (1680), Rennes (1767), Toulouse (1702 et 1706) : AD Cher, D 5, fos 154-161 ; AM Nantes, GG 667, non paginé ; Patrick Ferté, L'Université de Cahors au XVIII siècle, 1700-1751. Le coma universitaire au siècle des Lumières, Saint-Sulpice-la-Pointe/Toulouse, impr. Fournié, 1975, p. 221-226. 


\section{III - Les décalages du « processus de civilisation »}

Ce qui frappe dans la description qui vient d'être faite, c'est combien elle s'écarte peu de celle d'Ariès, en dépit de la bien plus grande abondance de documentation consultée. Même si celui-ci n'avait, en 1960, pas pleinement saisi les difficultés des facultés à contrôler les étudiants au XVIII ${ }^{\mathrm{e}}$ siècle, sa vision de l'évolution de la discipline dans les collèges reste valide.

C'est également à juste titre qu'Ariès affirme que les élèves français se sont " civilisés " bien plus tôt que leurs homologues anglais. Toute la documentation accumulée au cours des quarante dernières années confirme son idée qu'il faut attendre le milieu du XIX ${ }^{\mathrm{e}}$ siècle pour voir la paix régner dans les salles de classe anglaises. Encore au début de ce siècle, les élèves des grandes public schools anglaises (Eton, Harrow, Winchester, etc.) sont irrespectueux, indisciplinés et prompts à la rébellion. La vie quotidienne dans ces internats semble être une suite ininterrompue de bizarres cérémonies initiatiques, de combats et de bizutages, qui se terminent parfois par la mort d'un élève. La " rôtissoire ", qui consiste à maintenir un garçon le dos au feu jusqu'à ce qu'il hurle de douleur, est un passe-temps habituel, de même que les combats de boxe. Quand un élève d'Eton, Stephen Terry, se bat avec son condisciple Jack Musters, à la fin du XVIII ${ }^{\text {e }}$ siècle, les deux adversaires se bourrent mutuellement de coups avec tant d'acharnement qu'ils sont à peine reconnaissables quand ils se déclarent satisfaits $^{31}$. La conduite des étudiants d'Oxford et de Cambridge est tout aussi répréhensible. Théoriquement, chaque étudiant est confié à un tuteur personnel, qui est censé l'empêcher de nuire en même temps qu'organiser ses études. En réalité, beaucoup de ces tuteurs négligent leur charge : pendant toute l'époque des Lumières, ils laissent les étudiants plus jeunes rôder dans les rues, où ils se livrent à des actes pendables, aller dans les tavernes et fréquenter les prostituées. Le vol de cadavres à des fins de dissection, comme cela se produit en 1730 lorsque la famille livre bataille aux étudiants pour la possession du mort, est le moindre de leurs forfaits ${ }^{32}$.

Ce qu'Ariès n'a pas cherché à expliquer, c'est la cause de ce décalage, qui est désormais nettement plus claire à nos yeux. Le " retard " apparent de l'Angleterre

31 Anthony Fletcher, Growing up in England. The Experience of Childhood 1600-1914, New Haven (Conn.), Yale University Press, 2008, chap. 13.

32 Graham Midgley, University Life in Eighteenth-Century Oxford, New Haven (Conn.), Yale University Press, 1996, chap. 11 (en particulier p. 146-147). 
à policer les étudiants peut être attribué, pour une large part, à son protestantisme, qui a rendu plus difficile la création des conditions nécessaires à un contrôle attentif et réfléchi des adolescents. Il n'y avait pas d'ordres religieux dans l'Angleterre protestante, donc pas de réservoir de maîtres capables et organisés de façon adéquate. En France, les élèves des collèges jésuites gardent le même régent quand ils montent de classe en classe, et l'enseignement de ce dernier est supervisé par ses supérieurs. De plus, il n'y a pas seulement continuité à l'intérieur des collèges jésuites, mais aussi standardisation à l'intérieur du réseau des collèges : des élèves changeant d'établissement ne se retrouvent pas dans un environnement totalement étranger. Dans les grammar schools anglaises, en revanche, les maîtres sont souvent peu capables et les ressources maigres : un enseignant est généralement quelqu'un qui manque des capacités et des relations nécessaires pour obtenir une cure dans une paroisse. Le système est, de plus, extrêmement compétitif et instable : le salaire est bas et il est facile d'attirer les maîtres vers un autre poste en plein milieu du trimestre, laissant ainsi leurs élèves à l'abandon.

Plus important encore, la culture religieuse protestante semble avoir joué contre l'invention pédagogique. Alors que le christianisme de la Réforme aussi bien que celui de la Contre-Réforme semblent tous deux fondés sur une conception augustinienne de l'humanité, l'Anglais calviniste ne voit dans le jeune aucune manifestation de présence d'une étincelle divine. Malgré l'appel à traiter les enfants avec plus de douceur lancé au XVIII ${ }^{\mathrm{e}}$ siècle par Locke et d'autres partisans d'une réforme pédagogique, ces sages propos n'ont toujours pas convaincu grand monde un siècle plus tard. Dans la classe comme au dehors, il faut battre les enfants pour les rendre obéissants. L'émulation ne joue donc qu'un rôle minime dans les écoles anglaises et la canne est employée de façon généreuse et en public. Dans les années 1820, à l'époque où Gladstone se trouve à Eton, l'école est encore dirigée d'une main de fer et son directeur, le sadique Thomas Keate, affectueusement appelé " le Fouetteur ". Ainsi, celui-ci administre délibérément une correction à un groupe d'élèves venus lui demander de faire leur confirmation. La violence aveugle du maître ne peut qu'encourager le désordre et la désobéissance : les élèves se soumettent à la violence physique comme à un fait naturel, mais ils ont aussi le sens de la justice naturelle ; ils prennent exemple sur l'autorité et traitent leurs maitres et leurs condisciples en conséquence. Les maîtres ne semblent pas non plus se soucier beaucoup des traitements que les élèves s'infligent les uns aux autres, du moment qu'on peut 
les réduire à l'obéissance par l'intimidation. Et jusqu'au début du XIX siècle, il est admis que la bagarre et le bizutage permettent aux garçons de devenir virils. C'est seulement par la suite que la formation de cette qualité passe par un combat ritualisé mené sur le terrain de sport : la violence est sublimée dans les sports d'équipe qui sont alors créés ${ }^{33}$.

On peut donc soutenir que l'élève respectueux et l'étudiant civilisé ont été produits par le monde de la Contre-Réforme : exemple intéressant d'une situation où protestantisme et modernité ne sont pas allés de pair. Bien entendu, cela peut n'être qu'un cas supplémentaire de "l'exception " anglaise. Il est possible que la jeunesse d'autres pays protestants ait été plus disciplinée. Nous avons de toute évidence besoin de davantage de recherches comparatives. De nombreuses données montrent, par exemple, que les étudiants des universités espagnoles étaient, dans la première moitié du XVII ${ }^{\mathrm{e}}$ siècle, tout aussi prompts à l'émeute que leurs prédécesseurs de la fin du Moyen Âge, ce qui semble contredire l'idée d'une parenté entre le catholicisme et le processus de civilisation. Mais cela peut n'être que le pur et simple reflet de la taille des universités castillanes à l'époque, surtout celle de Salamanque. Quand leurs effectifs ont diminué après 1650, notamment en raison de l'essor des collèges, les étudiants ont pu devenir plus dociles ${ }^{34}$. Peut-être les universités françaises ont-elles été pacifiées assez tôt parce que, à partir du règne des Bourbons, elles ont été de taille réduite, même celle de Paris ${ }^{35}$. Il n'est, pour l'instant, pas possible de conclure. Cet article aura atteint son but s'il a convaincu

33 Le premier directeur de public school à avoir réformé la sienne est Thomas Arnold, à Rugby, au cours des années 1830, mais il a eu du mal à imposer sa vision "douce " de l'éducation chrétienne : voir J. A. Mangan, "Social Darwinianism and Upper Class Education in Late Victorian and Edwardian England”, in J.A. Mangan, James Walvin (dir.), Manliness and Morality: Middle-class masculinity in Britain and America, 1800-1940, Manchester, Manchester University Press, 1987. La meilleure vue d'ensemble de l'évolution des public schools au XIX siècle est donnée par John Chandos, Boys together: English Public Schools 1800-1864, London, Hutchinson, 1984. Au cours des années 1830, la mauvaise discipline des public schools commença à subir la critique de romanciers victoriens tels que Thomas Hughes, l'auteur de Tom Brown's Schooldays (1857).

34 Richard L. Kagan, Students and Society in Early Modern Spain, Baltimore/London, John Hopkins University Press, 1974, p. 197 (graphique) ; Andrew Hegarty, "The Corporate University in the Age of Olivares: Salamanca, c. 1620-1640”, thèse, Université d'Oxford, 1998, passim.

35 Pour une estimation de leur taille, voir Laurence W. B. Brockliss, Higher Education..., op. cit., p. 17-18. Pour Paris, voir, du même, "Patterns of Attendance at the University of Paris, 1400-1800", Historical Journal, 21, 3, 1978, p. 503-544. 
ses lecteurs de la nécessité de prolonger le travail pionnier d'Ariès ${ }^{36}$ en approfondissant l'étude du contexte, institutionnel et culturel, dans lequel se sont transformés les modèles de comportement de la jeunesse à l'époque moderne.

Laurence BROCKLISS

Magdalen College, Oxford laurence.brockliss@history.ox.ac.uk

36 Malheureusement, la synthèse la plus récente sur l'université à l'époque moderne ne se penche pas sur la question du comportement des étudiants : Hilde de Ridder-Symoens (dir.), A History of the University of Europe, vol. II, Universities in Early Modern Europe (1500-1800), Cambridge, Cambridge University Press, 1996. 